Comparative Legilinguistics

vol. 30/2017

DOI : http://dx.doi.org/10.14746/cl.2017.30.5

\title{
LA NON-TRADUCTION DANS LA TRADUCTION JURIDIQUE. LE CAS DE L'UNION EUROPÉENNE
}

\author{
Anna WALICKA \\ Adam Mickiewicz University \\ Institute of Romance Studies \\ walickaa@amu.edu.pl
}

Résumé : Le présent article rapporte les résultats d'une étude sur corpus réalisée afin de définir la fréquence et l'envergure de la non-traduction intentionnelle dans les traductions juridiques. L'étude a été réalisée sur le corpus que forment les publications officielles de l'UE, majoritairement juridiques, accessibles sur le site eur-lex.europa.eu. Les exemples rapportés dans l'article sont tirés des versions anglaise, française et polonaise desdites publications.

Mots clés : Union européenne, traduction juridique, non-traduction.

\section{BRAK TLUMACZENIA W TLUMACZENIU PRAWNICZYM PRZYKLAD UNII EUROPEJSKIEJ}

Streszczenie: Niniejszy artykuł zawiera opis wyników badania korpusowego mającego na celu ustalenie częstotliwości i zakresu stosowania techniki tłumaczeniowej polegającej na zamierzonym nietłumaczeniu wybranych elementów tekstu wyjściowego w tekstach prawnych i prawniczych. Analizę przeprowadzono w oparciu o korpus, jakim jest zbiór wielojęzycznych oficjalnych publi- 
kacji prawnych i prawniczych UE, dostępny na stronie eur-lex.europa.eu. Przytoczone $\mathrm{w}$ artykule przykłady pochodzą $\mathrm{z}$ angielskiej, francuskiej oraz polskiej wersji językowej tychże dokumentów.

Słowa kluczowe: Unia Europejska, thumaczenie prawnicze, nietłumaczenie.

\section{NON-TRANSLATION IN LEGAL TRANSLATION. THE EXAMPLE OF THE EU.}

Abstract: The article describes the results of the corpus studies to determine the frequency and scope of the translation strategy involving the deliberate non-translation of selected elements of the source text in legislative and legal texts. The analysis was based on the multilingual official legislative and legal publications of the EU available at eur-lex.europa.eu. The examples quoted in the article come from equipollent versions in English, French and Polish.

Key words: European Union, legal translation, non-translation.

\section{Introduction}

Dans le présent article, je veux proposer au lecteur une réflexion sur la présence et le rôle des éléments lexicaux intentionnellement non traduits. Plus particulièrement, l'article rapportera les résultats d'une étude sur corpus réalisée afin de déterminer l'envergure et la fréquence de la non-traduction intentionnelle de lexies traduisibles dans les textes juridiques. L'étude a été réalisée sur le corpus formé par l'ensemble des publications officielles de l'UE accessibles sur le site eur-lex.europa.eu.

Afin de nommer la technique analysée dans cette contribution, certains auteurs recourent à la notion de «non-traduisibilité » (voir Vaxelaire 2005, Humbley 2006). Néanmoins, dans cette contribution je parlerai de la «non-traduction » pour éliminer ainsi toute association possible de ce mot avec la notion d'intraduisibilité, et pour marquer ainsi nettement l'opposition existante entre la non-traduction, étant un acte intentionnel du traducteur, et l'intraduisibilité conçue comme un «caractère d'un énoncé auquel on ne peut faire correspondre aucun énoncé équivalent dans une autre langue» (Cormier, Delisle et Lee-Jahnke 1999: 47). Ce qui différencie la non-traduction de l'intraduisibilité, c'est donc le refus intentionnel et conscient du traducteur de traduire des éléments lexicaux traduisibles. 
Loin d'être une technique marginale, la non-traduction est stipulée comme règle «de traduction » notamment par la Commission européenne, le Conseil de l'Europe et le Centre Européen pour l'Enseignement supérieur de l'UNESCO (voir Kierzkowska 2011: 83). Les principes de la non-traduction formulés par ces organismes concernent principalement le domaine de l'éducation (ibidem). Conformément à ces principes, ne devraient pas être traduits, même pour des fins informatives, les noms de titres ou de grades universitaires ainsi que les noms d'institutions (telles que les noms d'écoles ou d'universités).

Or, les règles formulées par la Commission européenne, le Conseil de l'Europe et l'UNESCO ne sont pas des règles communément admises. Autant, certains accords internationaux réfutent en effet la traduction pour des fins informatives de ce type de lexique (voir Kierzkowska 2011: 83), autant d'autres admettent sa traduction littérale, justement pour des fins informatives. L'exemple de cette dernière pratique constitue entre autres les accords concernant le domaine de l'éducation conclus entre le gouvernement de la République de Pologne (RP) et le gouvernement de la République fédérale d'Allemagne ou le gouvernement de la RP et le gouvernement de l'Autriche (voir Kubacki $2013: 30)$.

Dans le contexte polonais, nous pouvons observer deux tendances opposées. D’un côté, Kodeks thumacza przysięgłego («Code du traducteur juré », désormais le «KTP») préconise la non-traduction absolue de ce type de lexique (Kierzkowska 2011: 83-85), de l'autre, d'aucuns se prononcent en faveur de la traduction des noms de grades universitaires par exemple (voir Kubacki $2013: 31$ ).

Un tel manque d'unanimité, tant au niveau international qu'au niveau national, constitue une première raison pour approfondir la problématique de la non-traduction et s'interroger sur l'attitude à adopter par rapport à la non-traduction face aux préconisations qui divergent. Une deuxième raison constitue le fait que les traductions réalisées par les services de traduction de différentes institutions européennes (aussi bien celles de l'UE que celles d'autres organisations européennes) sont souvent considérées comme une source de «droit dérivé » par nombre de traducteurs locaux.

Dans le contexte polonais, cette influence pour ce qui est de la non-traduction se manifeste entre autres à travers les prescriptions formulées à l'intention des traducteurs jurés (assermentés) polonais dans le «KTP » (Kierzkowska 2011), principalement à l'article 39. Autant les 
versions précédentes du « $\mathrm{KTP}$ » autorisaient une traduction pour des fins informatives, autant la version du Code de 2011 l'interdit et préconise explicitement dans le domaine de l'éducation les règles de la non-traduction élaborées par la Commission européenne, le Conseil de l'Europe et le CEPES de l'UNESCO (Kierzkowska 2013 : 23).

Qu'en est-il réellement dans la pratique de la traduction des institutions européennes? La non-traduction y est-elle une pratique fréquente, régulière ou est-elle une exception réservée à un domaine, à un certain type de lexique ou encore à un type de document particulier ? Quels facteurs motivent le recours à cette technique de traduction? Voici les questions qui constituent le point de départ pour la réflexion et l'analyse qui suivront.

Afin de répondre à ces questions, je compte procéder en deux étapes. Premièrement, j'estime utile de rappeler en ce lieu que l'intraduisibilité, telle qu'elle a été définie dans l'introduction, est considérée comme une exception (voir Cormier, Delisle et Lee-Jahnke 1999: 47). Le traducteur étant en mesure de traduire presque tout, la non-traduction absolue devrait être, théoriquement du moins, une pratique plus qu'exceptionnelle.

J'espère démontrer cette supposition en présentant et en analysant par la suite une trentaine d'exemples provenant des versions anglaise, française et polonaise des publications officielles de l'UE. Les exemples rapportés illustreront différentes techniques de traduction des lexies dites parfois «à fort contenu culturel» (Houbert 2005 : 97), c'est-à-dire des lexies qui se réfèrent à des notions propres à une culture, enracinées profondément dans cette culture, et qui, de ce fait, n'ont pas d'équivalent sémantique direct dans d'autres langues. C'est à cette catégorie qu'appartiennent les noms de titres ou de grades universitaires, les noms d'institutions, ou encore les appellations officielles des textes législatifs (ibidem). Par conséquent, ils sont les plus susceptibles de rester non traduits, ce qui motive leur choix pour les besoins de l'étude rapportée dans cette contribution.

D’une manière générale, la non-traduction est associée principalement avec les noms propres (NP) dont la non-traduction était souvent donnée comme critère de leur définition (Humbley 2006). Cependant, la pratique et la théorie divergent sur ce point, ce qui a été souvent souligné dans les travaux consacrés à ce sujet (Vaxelaire 2005) car de nombreux NP étaient et sont toujours traduits. Dans certaines situations 
c'est même obligatoire : l'obligation de la traduction des NP concerne surtout ces noms propres qui possèdent un équivalent reconnu. En ce qui concerne les toponymes, leur équivalent reconnu doit figurer obligatoirement dans les traductions certifiées conformes, soit avant le nom original (Kubacki 2012b : 135), soit après ce nom (Kierzkowska 2011, 2013).

Bien qu'à l'origine la non-traduction ait concerné principalement les noms propres, actuellement, telle que l'on observe dans les documents officiels de l'UE, et plus particulièrement dans les arrêts et les ordonnances de la CJUE, elle s'étend à une catégorie lexicale beaucoup plus large et très hétérogène. La non-traduction s'y applique notamment non seulement aux noms propres de la catégorie NP sans équivalent reconnu, mais aussi aux noms propres de la catégorie $N P$ avec un équivalent reconnu. Bien qu'il s'agisse toujours principalement des noms propres, dont les noms d'institutions (écoles, universités, ministères, juridictions nationales), également les noms de fonctions y figurent non traduits.

Ainsi, nous pouvons observer dans les publications officielles de l'UE la non-traduction absolue qui concerne :

a. les noms de juridictions nationales :

Exemple 1

(http://eur-lex.europa.eu/legal-content/EN-FR-PL/TXT/?uri=CELEX:62008CJ 0314\&from=PL)

\begin{tabular}{|c|c|c|}
\hline EN & FR & PL \\
\hline $\begin{array}{l}\text { In Case C-314/08, RE- } \\
\text { FERENCE for a prelimi- } \\
\text { nary ruling under Article } \\
234 \text { EC by the Woje- } \\
\text { wódzki Sąd Admini- } \\
\text { stracyjny w Poznaniu } \\
\text { (Poland). (...) }\end{array}$ & $\begin{array}{c}\text { Dans l'affaire C-314/08, } \\
\text { ayant pour objet une } \\
\text { demande de décision } \\
\text { préjudicielle au titre de } \\
\text { l'article } 234 \text { CE, intro- } \\
\text { duite par le Wojewódzki } \\
\text { Sąd Administracyjny w } \\
\text { Poznaniu (Pologne). (...) }\end{array}$ & $\begin{array}{c}\text { W sprawie C-314/08 } \\
\text { mającej za przedmiot } \\
\text { wniosek o wydanie, na } \\
\text { podstawie art. } 234 \mathrm{WE}, \\
\text { orzeczenia w trybie pre- } \\
\text { judycjalnym, złożony } \\
\text { przez Wojewódzki Sąd } \\
\text { Administracyjny } \\
\text { w Poznaniu (Polska). (...) }\end{array}$ \\
\hline
\end{tabular}

Exemple 2

(http://eur-lex.europa.eu/legal-content/EN-FR-PL/TXT/?uri=CELEX:62014CN 0250\&from=ENann\%C3\%A9e:\%202014)

\begin{tabular}{|c|c|c|}
\hline EN & FR & PL \\
\hline $\begin{array}{l}\text { Request for a preliminary } \\
\text { ruling from the Conseil } \\
\text { d'État (France) lodged on } \\
26 \text { May 2014 - Air } \\
\text { France - KLM v Minis- } \\
\text { tère des finances et des }\end{array}$ & $\begin{array}{l}\text { Demande de décision } \\
\text { préjudicielle présentée par } \\
\text { le Conseil d'État (France) } \\
\text { le } 26 \text { mai } 2014 \text { - Air } \\
\text { France - KLM/Ministère } \\
\text { des finances et des }\end{array}$ & $\begin{array}{l}\text { Wniosek o wydanie orze- } \\
\text { czenia w trybie prejudy- } \\
\text { cjalnym złożony przez } \\
\text { Conseil d'État (Francja) } \\
\text { w dniu } 26 \text { maja } 2014 \text { r. - } \\
\text { Air France - KLM prze- }\end{array}$ \\
\hline
\end{tabular}




\begin{tabular}{|c|c|c|}
\hline comptes publics. & comptes publics. & $\begin{array}{c}\text { ciwko Ministère des fi- } \\
\text { nances et des comptes } \\
\text { publics. }\end{array}$ \\
\hline
\end{tabular}

Exemple 3

(http://eur-lex.europa.eu/legal-content/EN-FR-PL/TXT/?uri=CELEX:62015CN 0161\&from=FR)

\begin{tabular}{|c|c|c|}
\hline EN & FR & PL \\
\hline $\begin{array}{l}\text { Request for a prelimi- } \\
\text { nary ruling from the } \\
\text { Conseil d'État (Bel- } \\
\text { gium) lodged on } 9 \text { April } \\
2015 \text { - Abdelhafid } \\
\text { Bensada Benallal v } \\
\text { Belgian State. }\end{array}$ & $\begin{array}{l}\text { Demande de décision } \\
\text { préjudicielle présentée par } \\
\text { le Conseil d'État (Bel- } \\
\text { gique) le } 9 \text { avril } 2015 \text { - } \\
\text { Abdelhafid Bensada Be- } \\
\text { nallal/État belge. }\end{array}$ & $\begin{array}{c}\text { Wniosek o wydanie orze- } \\
\text { czenia w trybie prejudy- } \\
\text { cjalnym złożony przez } \\
\text { Conseil d'État (Belgia) } \\
\text { w dniu } 9 \text { kwietnia } 2015 \text { r. } \\
\text { - Abdelhafid Bensada } \\
\text { Benallal/Państwo Belgij- } \\
\text { skie. }\end{array}$ \\
\hline
\end{tabular}

\section{Exemple 4}

b. les noms de ministères :

(http://eur-lex.europa.eu/legal-content/EN-FR-PL/TXT/?uri=CELEX:62014CN 0250\&from $=\mathrm{EN} \% \mathrm{E} 2 \% 80 \% \mathrm{~A} 8 \mathrm{ann} \% \mathrm{C} 3 \% \mathrm{~A} 9 \mathrm{e}: \% 202014$ )

\begin{tabular}{|c|c|c|}
\hline EN & FR & PL \\
\hline $\begin{array}{l}\text { Request for a preliminary } \\
\text { ruling from the Conseil } \\
\text { d'État (France) lodged on } \\
26 \text { May } 2014-\text { Air } \\
\text { France - KLM v Minis- } \\
\text { tère des finances et des } \\
\text { comptes publics }\end{array}$ & $\begin{array}{l}\text { (...) Demande de décision } \\
\text { préjudicielle présentée par } \\
\text { le Conseil d'État (France) } \\
\text { le } 26 \text { mai } 2014 \text { - Air } \\
\text { France - } \\
\text { KLM/Ministère des } \\
\text { finances et des comptes } \\
\text { publics }\end{array}$ & $\begin{array}{l}\text { (...) Wniosek o wydanie } \\
\text { orzeczenia w trybie pre- } \\
\text { judycjalnym złożony } \\
\text { przez Conseil d'État } \\
\text { (Francja) w dniu } 26 \text { maja } \\
2014 \text { r. - Air France - } \\
\text { KLM przeciwko Mi- } \\
\text { nistère des finances et } \\
\text { des comptes publics }\end{array}$ \\
\hline $\begin{array}{l}\text { Defendant: Ministère des } \\
\text { finances et des comptes } \\
\text { publics }\end{array}$ & $\begin{array}{l}\text { Partie défenderesse: Mi- } \\
\text { nistère des finances et } \\
\text { des comptes publics }\end{array}$ & $\begin{array}{l}\text { Strona pozwana: Minis- } \\
\text { tère du budget, des } \\
\text { comptes publics et de la } \\
\text { fonction publique }\end{array}$ \\
\hline
\end{tabular}

c. les noms de fonctions :

Exemple 5

(http://eur-lex.europa.eu/legal-content/EN-FR-PL/TXT/?uri=CELEX:62008CJ 0314\&from=PL)

\begin{tabular}{|c|c|c|}
\hline EN & FR & PL \\
\hline $\begin{array}{l}\text { (...) in the proceedings } \\
\text { Krzysztof Filipiak } \\
\text { v. Dyrektor Izby Skar- } \\
\text { bowej w Poznaniu (...) }\end{array}$ & $\begin{array}{c}\text { (...) dans la procédure } \\
\text { Krzysztof Filipiak } \\
\text { contre } \\
\text { Dyrektor Izby Skarbo- } \\
\text { wej w Poznaniu (...) }\end{array}$ & $\begin{array}{c}\text { (...) w postępowaniu: } \\
\text { Krzysztof Filipiak } \\
\text { przeciwko } \\
\text { Dyrektorowi Izby Skar- } \\
\text { bowej w Poznaniu (...) }\end{array}$ \\
\hline
\end{tabular}


d. voire même les noms de formes de société : Exemple 6

(http://eur-lex.europa.eu/legal-content/FR-EN/TXT/?uri=CELEX:31987D0297 $\&$ from $=$ FR)

\begin{tabular}{|c|c|c|}
\hline EN & FR & PL \\
\hline $\begin{array}{l}\text { SOCIÉTÉ D’ÉNERGIE NU- } \\
\text { CLÉAIRE FRANCO_BELGE } \\
\text { DES ARDENNES (SENA) } \\
\text { - Société anonyme with a capital } \\
\text { of } 80 \text { million French francs - } \\
\text { Registered place of business : } 3 \\
\text { and 5, avenue de Friedland, Paris } \\
\text { 8e (RCS Paris B } 602043 \text { 838) }\end{array}$ & $\begin{array}{c}\text { SOCIÉTÉ D'ÉNERGIE NU- } \\
\text { CLÉAIRE FRANCO_BELGE } \\
\text { DES ARDENNES (SENA) - } \\
\text { Société anonyme au capital de } 80 \\
\text { millions de francs français - Siège } \\
\text { social : } 3 \text { et } 5 \text {, avenue de Frie- } \\
\text { dland, Paris } 8 \text { e (RCS Paris B } 602 \\
043 \text { 838) }\end{array}$ & $\begin{array}{l}\text { Traduction } \\
\text { inexistante }\end{array}$ \\
\hline $\begin{array}{l}\text { Article } 1 \\
\text { A société anonyme is hereby } \\
\text { formed by and between the own- } \\
\text { ers of the shares created below } \\
\text { and of the shares which may be } \\
\text { created in the future. }\end{array}$ & $\begin{array}{l}\text { Article premier } \\
\text { Il est formé par les présentes une } \\
\text { société anonyme qui existera } \\
\text { entre les propriétaires des actions } \\
\text { ci-après créées et de celles qui } \\
\text { viendraient à l'être par la suite. }\end{array}$ & $\begin{array}{l}\text { Traduction } \\
\text { inexistante }\end{array}$ \\
\hline
\end{tabular}

Les exemples ci-dessus présentent les lexies qui ne pourraient être considérées comme intraduisibles, du moins à la lumière de la définition de l'intraduisibilité proposée par la «Terminologie de la traduction » (Cormier, Delisle et Lee-Jahnke 1999 : 47), définition déjà citée dans l'introduction de cette contribution. Il convient de noter que les auteurs de l'ouvrage constatent que «l'intraduisibilité totale est exceptionnelle ». Elle ne peut être confondue avec la simple lacune qui peut être compensée par divers procédés de traduction dont l'application contribue à réduire la marge d'intraduisibilité (ibidem). En effet, l'intraduisibilité ne concerne qu'une marge minime du travail du traducteur. Le plus souvent les limites de la traduction sont atteintes « en traduction poétique en raison du lien unissant sonorités, images, rythmes et sens. Il en va de même de certains jeux de mots ou de tout effet sémantique indissociablement lié à l'exploitation des particularités formelles d'une langue » (ibidem). Les auteurs de l'ouvrage soulignent par ailleurs que «l'intraduisibilité ne s'applique pas au lexique ni à la terminologie. Lorsqu'un mot ou un terme n'a pas de correspondance dans une langue donnée, on a recours, selon le cas, à l'emprunt, au calque, à la création néologique ou à tout autre procédé pertinent » (ibidem : 47-48).

Aussi bien les noms de juridictions nationales que les noms de fonctions des exemples ci-dessus sont donc des noms traduisibles, 
quoique encrés profondément dans la culture d'un pays donné. De ce fait, de telles lexies sont parfois nommées par certains auteurs polonais « termes sans équivalent» - terminy bezekwiwalentowe (Pieńkos 1999, Krzywda 2014), ou « lexique sans équivalent»-leksyka bezekwiwalentowa (Płużyczka 2011). Ces appellations permettent de souligner le fait que l'on a affaire aux mots qui servent à exprimer les notions de la culture A qui n'existent pas à l'identique dans la culture B.

Cependant, comme le soulignent Pisarska et Tomaszkiewicz (1998), il ne s'agit pas de constater qu'il existe ou non un mot correspondant à un mot ou à une notion dans la langue source, donc un équivalent, mais il s'agit de constater qu'il existe ou non dans la culture de la langue cible une réalité à laquelle renvoie ce mot ou cette notion dans la langue source.

De même, Pieńkos souligne que le traducteur juridique doit avant tout identifier la fonction que remplit une lexie dans le texte source. Selon ce chercheur, traduire des textes juridiques c'est indiquer dans la langue cible un terme, un concept ou une institution dont la fonction est la même dans le texte source ou dont la définition dans le texte source est similaire (Pieńkos 1999).

Traduire c'est donc avant tout faire comprendre le sens d'un message, transmettre le sens d'un message dans une langue différente. Un procédé de traduction est ainsi défini comme "procédé de transfert linguistique des éléments de sens du texte de départ appliqué par le traducteur au moment où il formule une équivalence » (Cormier, Delisle et Lee-Jahnke 1999 : 64). Le processus de traduction est à son tour défini comme "opération intellectuelle par laquelle le traducteur établit des équivalences interlinguistiques» (ibidem). "Lors de cette opération complexe, le traducteur procède (...) à l'application de procédés de traduction, à la dissociation des langues en présence, à l'exploration des ressources de la (langue d'arrivée), au choix des moyens de réexpression et à la vérification de la pertinence des équivalences retenues » (ibidem). La théorie de la traduction confirme ainsi l'exceptionnalité de la non-traduction.

Le rejet de la non-traduction est visible également dans la pratique socio-professionnelle de la traduction. Il en témoigne entre autres le fait que même des lexies n'ayant pas leur équivalent notionnel dans d'autres cultures finissent tôt ou tard par en trouver un. Dans le contexte polonais, l'exemple en constitue le «RCS » français, terme qui ne fonctionnait même pas comme une entrée de dictionnaires franco-polonais 
pendant de longues années jusqu'à la parution du « Dictionnaire de la terminologie juridique français-polonais » de Machowska (2013). Une rareté des termes non traduits d'une manière régulière (tels que common law) prouve également que les traducteurs cherchaient et cherchent toujours à traduire des termes propres à une culture spécifique, une non-équivalence notionnelle n'étant pas un obstacle à leur traduction.

La pratique de la traduction réalisée par les services de traduction de différentes institutions de l'UE ne devrait pas s'éloigner de cette théorie. Pourquoi alors les lexies traduisibles provenant des exemples cités dans la première partie de l'article n'ont pas été traduites ? Premièrement, c'est le type de document qui en a décidé, et plus particulièrement la fonction y remplie par les lexies non traduites. La majorité des exemples ci-dessus proviennent ainsi des arrêts de la CJUE (sauf le dernier) dont les règles de rédaction définissent précisément la structure ainsi que l'emplacement des informations obligatoires spécifiques. Ainsi, au début de l'arrêt, dans sa partie introductive, sont rapportées les désignations des parties, considérées dans leur totalité comme des noms propres à part entière, et, de ce fait, non traduites ni accompagnées de quelconque traduction pour des fins informatives au début du texte. Les exemples ci-dessus proviennent justement de cette partie des arrêts. Cependant, une traduction pour des fins informatives apparaît très souvent plus loin dans le corps du texte. En voici quelques exemples :

\section{Exemple 7}

(http://eur-lex.europa.eu/legal-content/FR-EN-PL/TXT/?uri=CELEX:52010DC 0401\&from=PL)

\begin{tabular}{|c|c|c|}
\hline EN & FR & PL \\
\hline $\begin{array}{l}\text { 2. The reference has been } \\
\text { made in the context of } \\
\text { proceedings between Mr } \\
\text { Filipiak, a Polish national } \\
\text { who is subject to unlim- } \\
\text { ited tax liability in Poland, } \\
\text { and the Dyrektor Izby } \\
\text { Skarbowej w Poznaniu } \\
\text { (Director of the Poznań } \\
\text { Tax Chamber) ('the } \\
\text { Dyrektor') (...). }\end{array}$ & $\begin{array}{l}\text { 2. Cette demande a été } \\
\text { présentée dans le cadre } \\
\text { d'un litige opposant M. } \\
\text { Filipiak, ressortissant } \\
\text { polonais soumis à l'impôt } \\
\text { de manière illimitée en } \\
\text { Pologne, au Dyrektor Izby } \\
\text { Skarbowej w Poznaniu } \\
\text { (directeur de la chambre } \\
\text { fiscale de Poznań) (...). }\end{array}$ & 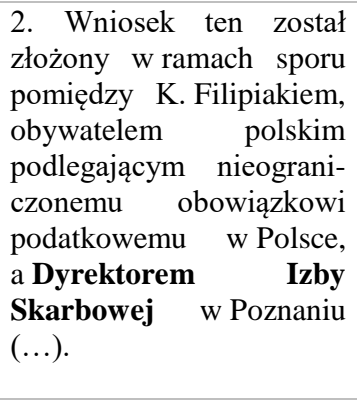 \\
\hline
\end{tabular}




\begin{tabular}{|c|c|c|}
\hline EN & FR & PL \\
\hline $\begin{array}{l}\text { 20. Mr Filipiak brought an } \\
\text { action against those deci- } \\
\text { sions before the Wo- } \\
\text { jewódzki Sąd Admin- } \\
\text { istracyjny w Poznaniu } \\
\text { (Regional Administra- } \\
\text { tive Court, Poznań) } \\
\text { (Poland) (...). }\end{array}$ & $\begin{array}{l}\text { 20. M. Filipiak a intro- } \\
\text { duit devant le } \\
\text { Wojewódzki Sąd Admi- } \\
\text { nistracyjny w Poznaniu } \\
\text { (tribunal administratif } \\
\text { de Poznań) des recours } \\
\text { contre ces décisions } \\
(\ldots) \text {. }\end{array}$ & $\begin{array}{l}\text { 20. K. Filipiak wniósł do } \\
\text { Wojewódzkiego Sądu } \\
\text { Administracyjnego } \\
\text { w Poznaniu skargi na te } \\
\text { decyzje }(\ldots)\end{array}$ \\
\hline \multicolumn{3}{|c|}{$\begin{array}{l}\text { Exemple } 9 \\
\text { (http://eur-lex.europa.eu/legal-content/EN-FR-PL/TXT/?uri=CELEX:62006TO } \\
\text { 0236\&from=PL) }\end{array}$} \\
\hline EN & FR & PL \\
\hline $\begin{array}{l}\text { (...) In constitutional } \\
\text { matters, the applicant has } \\
\text { the capacity to bring legal } \\
\text { proceedings or be a party } \\
\text { before the Bundesver- } \\
\text { fassungsgericht (Federal } \\
\text { Constitutional Court) } \\
\text { and the Landesverfas- } \\
\text { sungsgericht (Constitu- } \\
\text { tional Court of the Land } \\
\text { ). }\end{array}$ & $\begin{array}{r}\text { Pour les litiges co } \\
\text { tionnels, le requ } \\
\text { aurait la capacité } \\
\text { en justice ou d'être } \\
\text { devant le Bundes } \\
\text { sungsgericht (C } \\
\text { constitutionnelle } \\
\text { rale allemande) } \\
\text { Landesverfassun } \\
\text { richt (tribunal co } \\
\text { tionnel région }\end{array}$ & $\begin{array}{r}\text { (...)W odniesier } \\
\text { sporów konstytuc } \\
\text { posiada on zdolno } \\
\text { cesową oraz zdo } \\
\text { sądową przed B } \\
\text { sverfassungsge } \\
\text { (niemiecki fede } \\
\text { trybunal konstyt } \\
\text { oraz Landesve } \\
\text { sungsgericht (reg } \\
\text { trybunal konstytı }\end{array}$ \\
\hline \multicolumn{3}{|c|}{$\begin{array}{l}\text { Exemple } 10 \\
\text { (http://eur-lex.europa.eu/legal-content/EN-FR-PL/TXT/?uri=CELEX:62008CJ } \\
\text { 0314\&from=PL) }\end{array}$} \\
\hline EN & FR & PL \\
\hline $\begin{array}{l}\text { 7. Under Article } 188 \text { of } \\
\text { the Polish Constitution: } \\
\text { 'The Trybunal Konsty- } \\
\text { tucyjny [Polish Consti- } \\
\text { tutional Court] shall } \\
\text { adjudicate on the follow- } \\
\text { ing matters (...). }\end{array}$ & $\begin{array}{l}\text { 7. Aux termes de l'article } \\
188 \text { de la Constitution } \\
\text { polonaise: «Le Trybunal } \\
\text { Konstytucyjny [Tribunal } \\
\text { constitutionnel polonais] } \\
\text { statue sur (...). }\end{array}$ & $\begin{array}{l}\text { 7. Zgodnie z art. } 188 \\
\text { Konstytucji Rzeczypospo- } \\
\text { litej Polskiej: ,,Trybunal } \\
\text { Konstytucyjny orzeka } \\
\text { w sprawach (...). }\end{array}$ \\
\hline
\end{tabular}

Il convient de remarquer que l'usage des minuscules observé dans les traductions polonaises réalisées pour des fins informatives diffère de l'usage des traducteurs français et anglais. Dans les versions polonaises, on observe notamment les initiales en minuscule dans la traduction des deux noms de tribunaux : niemiecki federalny trybunat konstytucyjny et regionalny trybunat konstytucyjny (exemple 9), tandis que la version anglaise comporte les initiales en majuscule dans ces noms d'institutions, conformément aux règles de l'usage des majuscules propres à cette langue. Dans la version française à son tour, le traducteur recourt à ces deux procédés de traduction, en écrivant les initiales des 94 
équivalents de deux noms une fois en majuscule et l'autre fois en minuscule.

En ce qui concerne les documents de l'UE autres que les arrêts et les ordonnances de la CJUE, la non-traduction absolue y est un phénomène rarissime. Il en témoignent entre autres les deux exemples suivants provenant d'une décision de la Commission européenne de 2010 :

\section{Exemple 11}

(http://eur-lex.europa.eu/legal-content/FR-PL-EN/TXT/?uri=CELEX:32010D0 $373 \&$ fromTab-=ALL\&from=FR)

EN

(3) | Sabanci University, Tuzla/Istanbul, Turkey, McGill University, Montreal, Canada and Directorate Economic Service and Structural Reforms, Directorate General for Economic and Financial Affairs of the European Commission have to be regarded as (...).
FR

(3) | L'Université Sabanci de Tuzla/Istanbul, en Turquie, l'Université McGill de Montréal, au Canada, et la direction «Service économique et réformes structurelles» de la direction générale des affaires économiques et financières de la Commission européenne doivent être considérées comme (...).

Exemple 12

(http://eur-lex.europa.eu/legal-content/FR-PL-EN/TXT/?uri=CELEX:32010D0 $373 \&$ from-Tab=ALL\&from=FR)

\begin{tabular}{|l|l|l|}
\hline \multicolumn{1}{|c|}{ EN } & \multicolumn{1}{|c|}{ FR } & \multicolumn{1}{c|}{ PL } \\
\hline $\begin{array}{l}\text { University of Illinois at } \\
\text { Chicago (UIC), Chicago, } \\
\text { USA }\end{array}$ & $\begin{array}{l}\text { Université de l'Illinois à } \\
\text { Chicago (UIC), Chicago, } \\
\text { États-Unis }\end{array}$ & $\begin{array}{l}\text { Uniwersytet Illinois w } \\
\text { Chicago (UIC), Chicago, } \\
\text { Stany Zjednoczone Ame- } \\
\text { ryki }\end{array}$ \\
\hline
\end{tabular}

Les deux exemples ci-dessus montrent les divergences des procédés de traduction adoptés pour les noms d'universités dans la version polonaise de la décision de la CE. On y retrouve d'un côté les formes anglaises complètes des noms de l'Université Sabanci et de l'Université McGill. De l'autre côté, dans le même texte, le nom de l'Université de l'Illinois est rendu en polonais différemment, à savoir le nom commun « université » est cette fois-ci traduit vers le polonais.

Il semble que dès lors qu'un nom d'université contient un anthroponyme, le traducteur de la version polonaise décide de garder la version anglaise de ce nom, mais lorsque dans le nom de celle-ci figure 
un toponyme, le traducteur polonais rend le nom commun « université » par uniwersytet. Dès lors qu'un nom d'université comprend un toponyme ayant un équivalent reconnu, cet équivalent est rendu dans la version polonaise, comme en témoigne l'exemple $n^{\circ} 13$ de la même décision :

\section{Exemple 13}

(http://eur-lex.europa.eu/legal-content/FR-PL-EN/TXT/?uri=CELEX:32010D0 $373 \&$ from - Tab $=$ ALL \& from $=$ FR)

\begin{tabular}{|l|l|l|}
\hline \multicolumn{1}{|c|}{ EN } & \multicolumn{1}{|c|}{ FR } & \multicolumn{1}{|c|}{ PL } \\
\hline $\begin{array}{l}\text { Rady School of Manage- } \\
\text { ment at the University of } \\
\text { California, San Diego, } \\
\text { USA }\end{array}$ & $\begin{array}{l}\text { Rady School of Manage- } \\
\text { ment de l'Université de } \\
\text { Californie, San Diego, } \\
\text { États-Unis }\end{array}$ & $\begin{array}{l}\text { Szkoła } » \text { Rady School of } \\
\text { Management « na Uni- } \\
\text { wersytecie Kalifornij- } \\
\text { skim (University of Cali- } \\
\text { fornia), San Diego, Stany } \\
\text { Zjednoczone Ameryki }\end{array}$ \\
\hline
\end{tabular}

Dans la version française, les noms communs, qui composent les noms d'universités, sont régulièrement rendus en français. En ce qui concerne les toponymes, les exemples $n^{\circ} 11$ et 13 semblent confirmer le recours des traducteurs français à la même règle de traduction que celle appliquée dans les versions polonaises (qui est en même temps la règle de traduction préconisée par le KTP).

En ce qui concerne les noms de titres ou de grades, ils ne sont pas traduits même pour des fins informatives lorsque les institutions de l'UE adressent un avis de recrutement directement aux citoyens européens. Voici l'exemple d'un avis de recrutement du Parlement européen de 2014 :

\section{Exemple 14}

(http://eur-lex.europa.eu/legal-content/EN-FR-PL/TXT/?uri=CELEX:C2014/02 $6 \mathrm{~A} / 01 \&$ from $=\mathrm{PL}$ )

\begin{tabular}{|c|c|c|}
\hline EN & FR & PL \\
\hline $\begin{array}{l}\text { France | Maîtrise/MST } \\
\text { (maîtrise des sciences et } \\
\text { techniques)/MSG (maî- } \\
\text { trise des sciences de ges- } \\
\text { tion) | DEST (diplôme } \\
\text { d'études supérieures } \\
\text { techniques)/DRT (di- } \\
\text { plôme de recherche tech- } \\
\text { nologique) | DESS (di- } \\
\text { plôme d'études supé- } \\
\text { rieures spécialisées)/DEA }\end{array}$ & $\begin{array}{l}\text { France | Maîtrise/MST } \\
\text { (maîtrise des sciences et } \\
\text { techniques)/MSG (maî- } \\
\text { trise des sciences de ges- } \\
\text { tion) | DEST (diplôme } \\
\text { d'études supérieures } \\
\text { techniques)/DRT (di- } \\
\text { plôme de recherche tech- } \\
\text { nologique) | DESS (di- } \\
\text { plôme d'études supé- } \\
\text { rieures spécialisées)/DEA }\end{array}$ & $\begin{array}{l}\text { France | Maîtrise/MST } \\
\text { (maîtrise des sciences et } \\
\text { techniques)/MSG (maî- } \\
\text { trise des sciences de ges- } \\
\text { tion) | DEST (diplôme } \\
\text { d'études supérieures } \\
\text { techniques)/DRT (di- } \\
\text { plôme de recherche tech- } \\
\text { nologique) | DESS (di- } \\
\text { plôme d'études supé- } \\
\text { rieures spécialisées)/DEA }\end{array}$ \\
\hline
\end{tabular}




\begin{tabular}{|c|c|c|}
\hline $\begin{array}{c}\text { (diplôme d'études appro- } \\
\text { fondies) | Master 1/Master } \\
2 \text { professionnel/Master } 2 \\
\text { recherche | Diplôme des } \\
\text { grandes écoles/Diplôme } \\
\text { d'ingénieur/Doctorat | } \\
\text { Licence }\end{array}$ & $\begin{array}{l}\text { (diplôme d'études appro- } \\
\text { fondies) | Master 1/Master } \\
2 \text { professionnel/Master } 2 \\
\text { recherche | Diplôme des } \\
\text { grandes écoles/Diplôme } \\
\text { d'ingénieur/Doctorat | } \\
\text { Licence }\end{array}$ & $\begin{array}{l}\text { (diplôme d'études appro- } \\
\text { fondies) | Master 1/Master } \\
2 \text { professionnel/Master } 2 \\
\text { recherche | Diplôme des } \\
\text { grandes écoles/Diplôme } \\
\text { d'ingénieur/Doctorat | } \\
\text { Licence }\end{array}$ \\
\hline $\begin{array}{c}\text { Polska | Magi- } \\
\text { ster/Magister inżynier | }\end{array}$ & $\begin{array}{l}\text { Polska | Magi- } \\
\text { ster/Magister inżynier | }\end{array}$ & $\begin{array}{l}\text { Polska | Magi- } \\
\text { ster/Magister inżynier | }\end{array}$ \\
\hline $\begin{array}{l}\text { Dyplom doktora | Licen- } \\
\text { cjat/Inżynier }\end{array}$ & $\begin{array}{l}\text { Dyplom doktora | Licen- } \\
\text { cjat/Inżynier }\end{array}$ & $\begin{array}{l}\text { Dyplom doktora | Licen- } \\
\text { cjat/Inżynier }\end{array}$ \\
\hline
\end{tabular}

Dans d'autres types de documents, il est extrêmement rare de rencontrer les noms de titres ou de grades non traduits. Il en va de même des noms de diplômes universitaires composés évidemment de noms de titres ou de grades universitaires. D'une manière générale, les traducteurs recourent alors soit uniquement à leurs équivalents fonctionnels dans différentes langues traduites (exemple $\mathrm{n}^{\circ} 15$ ci-dessous), soit aux équivalents fonctionnels accompagnés des désignations originales, comme dans l'exemple $\mathrm{n}^{\circ} 16$ :

\section{Exemple 15}

(http://eur-lex.europa.eu/legal-content/EN-FR-PL/TXT/?uri=CELEX:12003TN $02 / 02 / \mathrm{C} 4 \&$ from=FR)

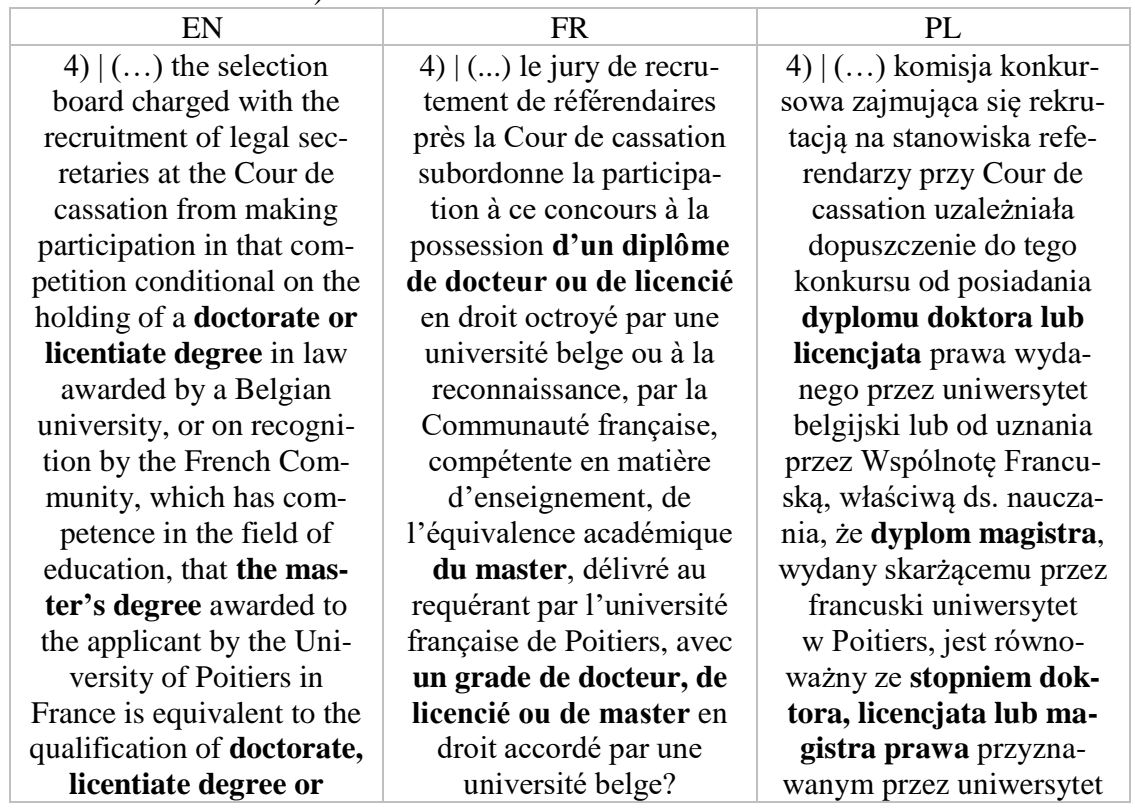




\begin{tabular}{|c|c|c|}
\hline $\begin{array}{l}\text { master's degree in law } \\
\text { awarded by a Belgian } \\
\text { university? }\end{array}$ & & belgijski? \\
\hline \multicolumn{3}{|c|}{$\begin{array}{l}\text { Exemple } 16 \\
\text { (http://eur-lex.europa.eu/legal-content/EN-FR-PL/TXT/?uri=CELEX:12003TN } \\
\text { 02/02/C4\&from=FR) }\end{array}$} \\
\hline EN & FR & PL \\
\hline $\begin{array}{c}\text { (s) in Lithuania: } \\
\text { - the engineer archi- } \\
\text { tect/architect diplomas } \\
\text { awarded by Kauno } \\
\text { politechnikos institutas } \\
\text { until } 1969 \text { (inžinierius } \\
\text { architektas/architektas), } \\
\text { - the architect/bachelor of } \\
\text { architecture/master of } \\
\text { architecture diplomas } \\
\text { awarded by (...) (archi- } \\
\text { tektas/architektûros } \\
\text { bakalau- } \\
\text { ras/architektûros magi- } \\
\text { stras), }\end{array}$ & $\begin{array}{c}\text { s) en Lituanie: } \\
\text { - les diplômes } \\
\text { d'ingénieur archi- } \\
\text { tecte/architecte délivrés } \\
\text { par le Kauno politechni- } \\
\text { kos institutas jusqu'en } \\
1969 \text { (inžinierius ar- } \\
\text { chitektas/architektas), } \\
\text { - les diplômes } \\
\text { d'architecte/diplômes de } \\
\text { niveau licence et maîtrise } \\
\text { en architecture délivrés } \\
\text { par (...) (architek- } \\
\text { tas/architektûros baka- } \\
\text { lauras/architektûros } \\
\text { magistras), }\end{array}$ & $\begin{array}{c}\text { s) na Litwie: } \\
\text { - dyplomy inżyniera } \\
\text {-architekta/architekta } \\
\text { wydawane przez Kauno } \\
\text { politechnikos institutas do } \\
1969 \text { r. (inžinierius ar- } \\
\text { chitektas/architektas), } \\
\text { - dyplomy architek- } \\
\text { ta/licencjacki architektu- } \\
\text { ry/magisterski architek- } \\
\text { tury wydawane przez (...) } \\
\text { (architek- } \\
\text { tas/architektûros baka- } \\
\text { lauras/architektûros } \\
\text { magistras) }\end{array}$ \\
\hline
\end{tabular}

Pour ce qui est des noms de postes universitaires, ces noms sont généralement traduits et parfois accompagnés de leurs versions originales. Ainsi, profesor uniwersytecki (wyktadajacy na uniwersytecie, profesor nadzwyczajny) et profesor zwyczajny sont généralement des équivalents fonctionnels polonais des noms de postes de "professeur des universités » (ou « professeur associé ») et de " professeur (de droit) ordinaire » (exemples 17-19). Le terme lektor est un équivalent polonais du poste de « lecteur de langue étrangère » (exemples $n^{\circ} 20$ et 21$)$ :

\section{Exemple 17}

(http://eur-lex.europa.eu/legal-content/EN-FR-PL/TXT/?uri=CELEX:62001CJ 0224\&from=PL)

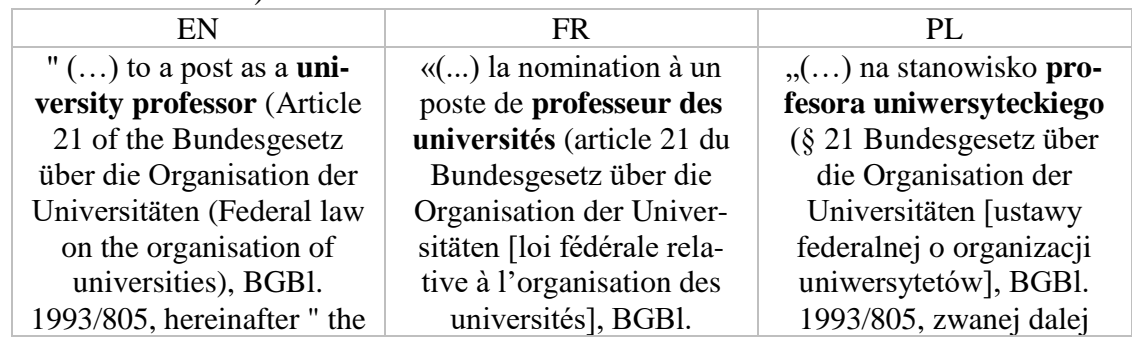


UOG 1993" ) or as an ordinary professor of universities or of an institution of higher education.
1993/805, dénommé UOG 1993') ou de professeur ordinaire des universités ou d'un établissement d'enseignement supérieur.»

Exemple 18

(http://eur-lex.europa.eu/legal-content/EN-FR-PL/TXT/?uri=CELEX:62003CO 0250\&from $=$ PL)

\begin{tabular}{|c|c|c|}
\hline EN & FR & PL \\
\hline $\begin{array}{c}\text { 4. (...) of at least the } \\
\text { standing of a counsellor of } \\
\text { the Court of Appeal and } \\
\text { one full or associate } \\
\text { professor of law at a } \\
\text { university or an institute } \\
\text { of higher education. }\end{array}$ & $\begin{array}{c}\text { 4. (...) non inférieure à } \\
\text { celle de conseiller à la } \\
\text { Cour d'appel, et un pro- } \\
\text { fesseur de droit ordi- } \\
\text { naire ou un professeur } \\
\text { associé enseignant dans } \\
\text { une université ou un } \\
\text { institut d'enseignement } \\
\text { supérieur. }\end{array}$ & $\begin{array}{c}\text { 4. (...) w stopniu nie niż- } \\
\text { Corte d'appello oraz } \\
\text { profesora zwyczajnego } \\
\text { lub profesora wyklada- } \\
\text { jącego na uniwersytecie } \\
\text { lub w instytucie studiów }\end{array}$ \\
\hline
\end{tabular}

Exemple 19

(http://eur-lex.europa.eu/legal-content/EN-FR-PL/TXT/?uri=CELEX:62011CJ 0159\&from $=$ PL)

\begin{tabular}{|l|l|l|}
\hline \multicolumn{2}{|c|}{ EN } & \multicolumn{1}{|c|}{ FR } \\
\hline $\begin{array}{l}\text { cost of an associate } \\
\text { professor }\end{array}$ & $\begin{array}{l}\text { - coût d'un professeur } \\
\text { associé }\end{array}$ & $\begin{array}{l}\text { - koszty profesora nad- } \\
\text { zwyczajnego }\end{array}$ \\
\hline
\end{tabular}

Exemple 20

(http://eur-lex.europa.eu/legal-content/EN-FR-IT/TXT/?uri=CELEX:61991CJ0 259\&from=IT)

\begin{tabular}{|c|c|c|}
\hline EN & FR & IT \\
\hline $\begin{array}{c}6(\ldots) \text { the employment } \\
\text { relationship between } \\
\text { universities and foreign } \\
\text { language assistants } \\
\text { where there is, in princi- } \\
\text { ple, no such limit with } \\
\text { regard to other workers. }\end{array}$ & $\begin{array}{c}6(\ldots) \text { la relation de travail } \\
\text { entre les universités et les } \\
\text { lecteurs de langue } \\
\text { étrangère, alors qu' une } \\
\text { telle limite n' existe pas, } \\
\text { en principe, en ce qui } \\
\text { concerne les autres tra- } \\
\text { vailleurs. }\end{array}$ & $\begin{array}{c}6 \text { (...) rapporto di lavoro } \\
\text { fra le Università e i lettori } \\
\text { di lingua straniera, } \\
\text { mentre tale limitazione } \\
\text { non esiste, in via di prin- } \\
\text { cipio, per quanto riguarda } \\
\text { gli altri lavoratori. }\end{array}$ \\
\hline
\end{tabular}

Exemple 21

(http://eur-lex.europa.eu/legal-content/EN-FR-PL/TXT/?uri=CELEX:62007CJ 0276\&from=IT)

\begin{tabular}{|c|c|c|}
\hline EN & FR & PL \\
\hline $\begin{array}{l}\text { 4. Foreign nationals may } \\
\text { be appointed (...) to spe- } \\
\text { cial posts of for- } \\
\text { eign-language and for- } \\
\text { eign-literature and } \\
\text {-language assistant in } \\
\text { addition to tenured assis- }\end{array}$ & $\begin{array}{l}\text { 4. (...) outre les postes de } \\
\text { lecteurs titulaires, des } \\
\text { charges de cours en tant } \\
\text { que lecteurs de langue et } \\
\text { de littérature et langue } \\
\text { étrangère peuvent être } \\
\text { confiées à des ressortis- }\end{array}$ & $\begin{array}{c}\text { 4. (...) można powierzyć } \\
\text { specjalne obowiązki } \\
\text { lektora języka obcego } \\
\text { oraz lektora literatury } \\
\text { i języka obcego, także } \\
\text { w uzupełnieniu stanowisk } \\
\text { lektora mianowanego. }\end{array}$ \\
\hline
\end{tabular}


tant posts. (...) sants étrangers (...).

$(\ldots)$

La non-traduction absolue peut concerner seules les lexies qui n'ont pas leur équivalent fonctionnel dans une langue donnée. D'une manière générale, le nom du poste de " maître de conférence » n'est pas ainsi traduit dans les versions anglaises, ce pays n'ayant pas dans les structures de ses universités un poste aux fonctions et responsabilités équivalentes.

\section{Exemple 22}

(http://eur-lex.europa.eu/legal-content/EN-FR/TXT/?uri=CELEX:61993CJ004 $7 \&$ from=EN)

EN

Commission of the European

Communities, represented by

Marie Wolfcarius, of its Legal

Service, and Théophile

Margellos, Avocat, Maître de

Conférences at the University of Picardie (...).
FR

Commission des Communautés européennes, représentée par

Mme Marie Wolfcarius, membre du service juridique, et M. Théophile Margellos, avocat, maître de conférences à l' université de Picardie (...).
PL

Traduction inexistante

Néanmoins, même dans ce cas nous pouvons rencontrer des tentatives de la traduction d'un tel terme, comme en témoignent la version anglaise de l'exemple 23 et la version polonaise des exemples $n^{\circ} 24$ et 25 :

\section{Exemple 23}

(http://eur-lex.europa.eu/legal-content/EN-FR-IT/TXT/?uri=CELEX:61989TJ0 $160 \&$ from $=$ FR)

EN

51 (...) According to the applicant his university title should have been translated as "professeur agrégé permanent"

[tenured professor] and not "maître de conférences" [reader]; (...).
FR

51 (...) Selon lui, d' une part, il aurait fallu traduire son titre universitaire par "professeur agrégé permanent" et non par "maître de conférence" (...)
$\mathrm{Pl}$

Traduction inexistante

\section{Exemple 24}

(http://eur-lex.europa.eu/legal-content/EN-FR-PL/TXT/?uri=CELEX:62009CC 0250\&from $=$ FR)

\begin{tabular}{|c|c|c|}
\hline EN & FR & PL \\
\hline $\begin{array}{c}\text { 10. (..) and in the case of } \\
\text { professors, lecturers and }\end{array}$ & $\begin{array}{c}\text { 10. (...) et pour les profes- } \\
\text { seurs, les maîtres de }\end{array}$ & $\begin{array}{c}10)(\ldots) \text { a w odniesieniu } \\
\text { do profesorów, docentów }\end{array}$ \\
\hline
\end{tabular}


level I and II assistants, and holders of doctorates in science; $(\ldots)$ conférence et les assistants des niveaux I et II et les docteurs en sciences i starszych wykładowców

I i II stopnia oraz doktorów nauk $(\ldots)$

(...)

Exemple 25

(http://eur-lex.europa.eu/legal-content/EN-FR-PL/TXT/?uri=CELEX:62006CC 0201\&from $=$ FR)

\begin{tabular}{|c|c|c|}
\hline EN & FR & PL \\
\hline $\begin{array}{c}\text { (...) according to I. Baldi } \\
\text { (Lecturer at the University } \\
\text { of Bordeaux) (...) }\end{array}$ & $\begin{array}{c}\text { (...) selon Baldi, I., } \\
\text { (maître de conférence à } \\
\text { l'université de Bordeaux) }\end{array}$ & $\begin{array}{c}\text { (...) według I. Baldiego } \\
\text { (wykladowcy na Uni- } \\
\text { wersytecie w Bordeaux) }\end{array}$ \\
\hline
\end{tabular}

Les exemples 23-25 démontrent en même temps les incohérences dans la traduction d'une même lexie dans les versions anglaise (lecturer versus reader) et polonaise (docent versus wyktadowca).

Un manque de cohésion est visible à son tour dans les quatre exemples suivants provenant de la Recommandation de la CE concernant l'Etat de droit en Pologne. Aussi bien le nom du Tribunal constitutionnel (exemple 26) que le nom de la chambre basse du parlement polonais (Sejm) y ont été traduits, quoique dans ce dernier cas la version française recourt à trois procédés différents de la traduction de ce mot rendu en français soit par la «Diète », soit par Sejm sans explication en français, soit par Sejm avec son équivalent français «Diète » entre parenthèses.

\section{Exemple 26}

(http://eur-lex.europa.eu/legal-content/EN-FR-PL/TXT/?uri=CELEX:32016H1 $374 \&$ from $=$ PL)

\begin{tabular}{|c|c|c|}
\hline EN & FR & PL \\
\hline $\begin{array}{l}\text { (8) | In November 2015, } \\
\text { the Commission became } \\
\text { aware of an ongoing } \\
\text { dispute in Poland con- } \\
\text { cerning in particular the } \\
\text { composition of the Con- } \\
\text { stitutional Tribunal, as } \\
\text { well as the shortening of } \\
\text { the mandates of its current } \\
\text { President and } \\
\text { Vice-President. The Con- } \\
\text { stitutional Tribunal } \\
\text { rendered two judgments } \\
\text { on this matter, on } 3 \text { and } 9\end{array}$ & $\begin{array}{l}\text { (8)|En novembre 2015, la } \\
\text { Commission a eu con- } \\
\text { naissance d'un différend } \\
\text { en cours en Pologne, au } \\
\text { sujet notamment de la } \\
\text { composition du Tribunal } \\
\text { constitutionnel, ainsi que } \\
\text { du raccourcissement de la } \\
\text { durée des mandats de ses } \\
\text { présidents et } \\
\text { vice-président actuels. Le } \\
\text { Tribunal constitutionnel } \\
\text { a rendu deux décisions sur } \\
\text { cette question, les } 3 \text { et }\end{array}$ & $\begin{array}{c}\text { (8) | W listopadzie } 2015 \text { r. } \\
\text { Komisja dowiedziała się o } \\
\text { trwającym w Polsce spo- } \\
\text { rze, dotyczącym w szcze- } \\
\text { gólności składu Trybu- } \\
\text { nału Konstytucyjnego, } \\
\text { jak również skrócenia } \\
\text { kadencji obecnego prezesa } \\
\text { i wiceprezesa Trybunału. } \\
\text { Trybunal Konstytucyjny } \\
\text { wydał dwa wyroki w tej } \\
\text { sprawie - w dniach } 3 \text { i } 9 \\
\text { grudnia } 2015 \text { r. }\end{array}$ \\
\hline
\end{tabular}




\begin{tabular}{|c|c|c|}
\hline December 2015. & 9 décembre 2015. & \\
\hline \multicolumn{3}{|l|}{ Exemple 27} \\
\hline $\begin{array}{l}\text { (9) | On } 22 \text { December } \\
\text { 2015, the Sejm adopted a } \\
\text { law amending the law on } \\
\text { the Constitutional Tribu- } \\
\text { nal, which concerns the } \\
\text { functioning of the Tribu- } \\
\text { nal as well as the inde- } \\
\text { pendence of its judges (6). }\end{array}$ & $\begin{array}{c}\text { (9) Le } 22 \text { décembre } 2015 \text {, } \\
\text { le Sejm a adopté une loi } \\
\text { modifiant la loi sur le } \\
\text { Tribunal constitutionnel, } \\
\text { qui concerne le fonction- } \\
\text { nement du Tribunal ainsi } \\
\text { que l'indépendance de ses } \\
\text { juges (6) }\end{array}$ & $\begin{array}{l}\text { (9) | W dniu } 22 \text { grudnia } \\
2015 \text { r. Sejm uchwalił } \\
\text { ustawę o zmianie ustawy } \\
\text { o Trybunale Konstytu- } \\
\text { cyjnym, która dotyczy } \\
\text { funkcjonowania Trybuna- } \\
\text { łu oraz niezawisłości } \\
\text { sędziów Trybunału (6). }\end{array}$ \\
\hline \multicolumn{3}{|l|}{ Exemple 28} \\
\hline $\begin{array}{c}(27) \mid(\ldots) \text { In the course of } \\
\text { April an expert group was } \\
\text { composed in the Sejm to } \\
\text { help prepare a new law on } \\
\text { the Constitutional Tribu- } \\
\text { nal. }\end{array}$ & $\begin{array}{c}\text { (27) } \mid \text { (...) Dans le courant } \\
\text { du mois d'avril, un groupe } \\
\text { d'experts a été constitué } \\
\text { au sein de la Diète pour } \\
\text { contribuer à l'élaboration } \\
\text { d'une nouvelle loi relative } \\
\text { au Tribunal constitution- } \\
\text { nel. }\end{array}$ & $\begin{array}{c}\text { (27) } \mid(\ldots) \text { W kwietniu w } \\
\text { Sejmie utworzono zespół } \\
\text { ekspertów mający pomóc } \\
\text { przy opracowywaniu } \\
\text { nowej ustawy o Trybunale } \\
\text { Konstytucyjnym. }\end{array}$ \\
\hline \multicolumn{3}{|l|}{ Exemple 29} \\
\hline $\begin{array}{l}\text { 15. | The Commission } \\
\text { furthermore notes that } \\
\text { also the Venice Commis- } \\
\text { sion considers that (...) } \\
\text { and 'therefore calls on all } \\
\text { State organs and notably } \\
\text { the Sejm to fully respect } \\
\text { and implement the judg- } \\
\text { ments' }(23) \text {. }\end{array}$ & $\begin{array}{l}\text { 15. | La Commission fait } \\
\text { remarquer en outre que } \\
\text { (...) et «appelle par con- } \\
\text { séquent l'ensemble des } \\
\text { organes de l'État et no- } \\
\text { tamment le Sejm [la } \\
\text { Diète] à respecter et à } \\
\text { exécuter pleinement les } \\
\text { décisions» (23). }\end{array}$ & $\begin{array}{l}\text { 15. | Komisja pragnie } \\
\text { ponadto zauważyć, że } \\
\text { Komisja Wenecka rów- } \\
\text { nież jest zdania, iż (...) i } \\
\text { „wzywa zatem wszystkie } \\
\text { organy państwowe, a w } \\
\text { szczególności Sejm, do } \\
\text { pełnego przestrzegania i } \\
\text { wykonywania orzeczeń } \\
\text { Trybunału” (23). }\end{array}$ \\
\hline
\end{tabular}

Le dernier exemple, provenant de l'arrêt de la CJUE déjà cité, montre que les traducteurs des différentes versions linguistiques des publications officielles de l'UE proposent des équivalents fonctionnels également pour les titres des publications juridiques nationales les plus importantes.

\section{Exemple 30}

(http://eur-lex.europa.eu/legal-content/EN-FR-PL/TXT/?uri=CELEX:62008CJ 0314\&from $=\mathrm{PL}-$ )

\begin{tabular}{|c|c|c|}
\hline EN & FR & PL \\
\hline $\begin{array}{c}\text { '1. After publication } \\
\text { thereof in the Journal of } \\
\text { Laws of the Republic of } \\
\begin{array}{c}\text { Poland ( Dziennik Ustaw } \\
\text { ), a ratified international }\end{array}\end{array}$ & $\begin{array}{c}\text { «1. Après sa publication } \\
\text { au Journal officiel de la } \\
\text { République de Pologne, } \\
\text { un traité ratifié fait partie } \\
\text { intégrante de l'ordre }\end{array}$ & $\begin{array}{c}\text { "1. Ratyfikowana umowa } \\
\text { międzynarodowa, po jej } \\
\text { ogłonzeniu w Dzienniku } \\
\text { Ustaw Rzeczypospolitej } \\
\text { Polskiej, stanowi część }\end{array}$ \\
\hline
\end{tabular}


agreement shall constitute part of the domestic legal order and shall be directly applicable (...). juridique national; il est directement applicable (...). krajowego porządku prawnego i jest bezpośrednio stosowana (...).

\section{Conclusion}

Il résulte clairement des exemples analysés ci-dessus qu'il n'existe que deux situations qui, aux yeux des traducteurs de l'UE, justifient le recours à la non-traduction. En effet, seules ne sont pas traduites les désignations des parties dans les arrêts et les ordonnances qui, à juste titre, se voient considérées comme des noms propres. Ceci d'ailleurs n'empêche pas les traducteurs de traduire les noms communs qui les composent dans le corps du texte pour des fins informatives. Ne sont non plus traduites ces lexies qui se réfèrent aux notions propres à une culture spécifique et qui n'existent pas dans d'autres cultures, «maître de conférence » semble en constituer un exemple convaincant. La non-traduction n'est pas ainsi une pratique de grande envergure au sein des services de traduction de l'UE. Au contraire, l'UE semble réaffirmer l'exceptionnalité de cette technique de traduction.

Il convient cependant de noter que les techniques de traduction observées dans les exemples analysés dans cette contribution ne sont pas parfois conséquentes et changent d'un texte à l'autre (traductions différentes du nom du poste de "professeur associé»), voire d'une équipe linguistique à l'autre (traduction versus non-traduction du mot « université »). Ceci semble témoigner d'un besoin toujours existant d'uniformisation des règles de traduction entre différentes équipes linguistiques. 


\section{Bibliographie}

Cormier, Monique C., Jean Delisle et Hannelore Lee-Jahnke (éds.). 1999. Terminologie de la traduction. Amsterdam; Philadelphia : J. Benjamins.

Houbert, Frédéric. 2005. Guide pratique de la traduction juridique. Anglais-français. Paris : La maison du dictionnaire.

Humbley, John. 2006. La traduction des noms d'institutions. Meta : journal des traducteurs 51, numéro 4 (décembre 2006), https://www.erudit.org/revue/meta/2006/v51/n4/014334ar.pdf (accès le 16 mai 2016).

Kierzkowska, Danuta. 2011. Kodeks tłumacza przysięgłego z komentarzem 2011. Warszawa: Wydawnictwo Translegis.

Kierzkowska, Danuta. 2013. Nazwy własne w tłumaczeniu dokumentów w świetle prawa polskiego i międzynarodowego. Lingua Legis 21: p. 16-25.

Krzywda, Joanna. 2014. Terminologia języka prawnego i strategie translalorskie w przekładach kodeksu spółek handlowych na jezyk niemiecki. Kraków : Wydawnictwo Uniwersytetu Jagiellońskiego.

Kubacki, Artur. 2012a. O problemach tłumaczenia poświadczonego polskich dokumentów szkolnych. Comparative Legilinguistics 9,

http://legilinguistics.amu.edu.pl/NUMERY/Comparative\%209 \%202012.pdf (accès le 11 avril 2016).

Kubacki, Artur. 2012b. Tłumaczenie poświadczone. Status, kształcenie, warsztat i odpowiedzialność tłumacza przysięgłego. Warszawa: Wolters Kluwer Business.

Kubacki, Artur. 2013. Problemy tłumaczenia niemieckich dokumentów szkolnych. Lingua Legis 21: p. 26-38.

Machowska, Aleksandra. 2013. Słownik terminologii prawniczej francusko-polski. Dictionnaire de la terminologie juridique français-polonais. Warszawa : Lex a Wolters Kluwer business.

Pieńkos, Jerzy. 1999. Podstawy juryslingwistyki: język w prawie prawo w języku. Warszawa : Oficyna Prawnicza MUZA SA.

Pisarska, Alicja, et Teresa Tomaszkiewicz. 1998. Współczesne tendencje przekładoznawcze: podręcznik dla studentów neofilologii. 
Poznań : Wydawnictwo Naukowe Uniwersytetu im. A. Mickiewicza.

Płużyczka, Monika, 2011. Wybrane trudności translacyjne a proces translodydaktyczny. In O nauczaniu i uczeniu się języka dla potrzeb zawodowych, S. Piotrowski (éd.), 88-97. Lublin : Wydawnictwo Werset.

Vaxelaire, Jean-Louis. 2005. Les Noms propres : une analyse lexicologique et historique. Paris : Champion. 